$\underline{\text { P-90 }}$

\title{
Preliminary Anti-Hypertension Studies of Orthosiphon Stamineus Standardised Ethanolic Extract
}

\author{
Armaghan Shafaei, Abdalrahim F.A. Aisha, Kameh Esmailli and Zhari Ismail ${ }^{*}$ \\ Department of Pharmaceutical Chemistry, School of Pharmaceutical Sciences, Universiti Sains Malaysia, Minden \\ 11800, Pulau Pinang, Malaysia; E-mails: Zhari Ismail, zhari@usm.my
}

Orthosiphon stamineus Benth. (Lamiaceae) or Misai Kucing is a medicinal plant grown in Southeast Asia particularly Malaysia and Indonesia. The leaves of the plant have been used for treatment of a wide range of ailments including diabetes, hypertension, gout and cancer. This study aims to investigate the anti-hypertension effect of standardized ethanolic extract of $O$. stamineus (OS-E) using in vitro and in vivo models. The in vitro anti-hypertensive effect was determined based on inhibitory effect on angiotensin converting enzyme (ACE) activity as a marker for anti-hypertension. However, the in vivo antihypertensive effect was investigated after oral administration of the extract on spontaneous hypertensive rats (SHR). The systolic blood pressure was measured before and after 14-days treatment by the tail cuff method. Ethanolic extract of $O$. stamineus was standardized based on the primary and secondary metabolites using HPLC, FT-IR, UV-VIS and gravimetric methods. In vitro testing showed modest inhibition of the ACE activity by $22.1 \%$, and the in vivo results in SHR rats showed significant reduction in the systolic blood pressure in 4 out of 6 treated rats after treatment period for 14 days. In conclusion, our preliminary results show blood pressure lowering effect of the OS-E extract which needs further investigation to explore the extract's mechanism of action.

Keyword: Orthosiphon stamineus, ethanol standardized extract, Anti-hypertension in vitro, in vivo. 